\title{
Unilateral torsion of fallopian tube at 32 of weeks of pregnancy followed by a normal delivery
}

Indira Upadhyaya, Richa Sharma

Paropakar Maternity and Women's Hospital

\begin{abstract}
A 24 year primi gravida at 32 weeks pregnancy presented with acute abdomen simulating torted ovarian tumor that underwent laparatomy/unilateral salpingectomy with the diagnosis of fallopian tube torsion is presented because of uneventful term vaginal delivery despite postoperative complication like gaped abdominal wound needing secondary suture.
\end{abstract}

Keywords: acute abdomen in pregnancy, acute abdomen due to fallopian tube torsion.

\section{Introduction}

Acute abdomen during third trimester of pregnancy presents as an obstetrical emergency and is usually diagnosed as a case of ovarian torsion or appendicitis. But the finding of twisted fallopian tube at laparotomy comes as a surprise, as the condition is uncommon though $12 \%$ cases are associated with pregnancy with the reported incidence of 1 in 1,500,000 women. ${ }^{1,2}$ In this context we are going to describe a pregnancy that continued to term resulting in a live birth after resection of a fallopian tube that had undergone torsion (unilateral salphingectomy) at 32 weeks pregnancy in a primigravida; despite postoperative morbidity consistent with abdominal wound infection leading to gaped wound needing resuture.

\section{Case}

A 24 year pregnant primigravida at 32 weeks presented with acute right sided lower abdomen pain. Clinically she was afebrile but tachycardia, dehydrated with normal blood pressure recording. Uterus corresponded to gestational age and fetal heart rate was regular. A diagnosis of urinary tract infection or twisted ovarian cyst was made. Preliminary urinary microscopic examination was normal and so were $\mathrm{CBC}$, serum biochemistry. In the next 24 hours, pain lower abdomen went on increasing without any drastic change in vital signs and there was no nausea or vomiting. However, generalized abdominal tenderness and guarding was observed in the right iliac fossae. Therefore USG abdomen was done that showed a singleton live baby at 32 weeks with normal liquor. An adnexal mass measuring $80 \times 60 \mathrm{~mm}$ was seen giving a suspicion of ovarian mass. Right renal pelvicalceal dilation was also noted. Making a diagnosis of right ovarian cyst torsion in pregnancy, emergency laparotomy was done. On the contrary, huge hydrosalpinx of the right tube was visualized which had undergone torsion twice on its axis with small fimbrial cyst. So partial right salpingectomy was performed. Left tube and ovaries were normal. Intra operative period was covered with i/ $\mathrm{m}$ inj. terbutalin $0.5 \mathrm{mg}$ to prevent premature uterine contraction and the latter was repeated six hours post operation.

The post operative period was complicated by gaping of the abdominal wound needing the application of secondary suture. She was discharge on 16th day of surgery after the removal of sutures. Fortunately, her pregnancy advanced smoothly ending in a spontaneous vaginal delivery of alive male baby at 38 weeks period of gestation. Her postnatal period was uneventful.

Correspondence

Indira Upadhyaya

MD, Obstetrician / Gynaecologist

Paropakar Maternity and Women's Hospital

Thapathali, Kathmandu

Mobile: 9851074598 


\section{Discussion}

Acute abdomen from torsion of fallopian tube has been described more than a century ago and a study of 201 cases of fallopian tube torsion has been published, although the exact number of fallopian tube torsion was not available. ${ }^{1}$ This rare condition is proposed to occur because of varied factors like anatomical abnormalities, like long mesosalphinx, tubal abnormalities, physiological abnormalities like abnormal peristalsis, tubal spasm, interstitial peristalsis and hemodynamic abnormalities. Other explanations are sudden body position changes (theory of Selheim), trauma, previous surgery or diseases and a gravid uterus. ${ }^{3-6}$ All of these which might lead fallopian tube to twist.

But because of the presence of sigmoid colon on the left and the slow venous drainage on the right with resultant congestion, torsion in right side is seen more frequently.

The most common presenting complaints is sudden cramping, intermittent lower abdomen pain that often radiates to the flank. Other associated symptoms include nausea, vomiting, increased frequency of micturation, loose motion, or difficult deafecation.

The body temperature is raised. WBC count may be normal or slightly elevated. There may be cervical tenderness and tender adnexae in pelvic examination. This case similarly presented with nausea and right lower abdomen pain but the count was rather normal for pregnancy.

In advanced pregnancy the ovaries move up and so it is difficult to be palpated vaginally. Moreover the adnexa are hidden underneath the pregnant uterus, so the USG has been very helpful. It is said that the torsion can be detected by color Doppler from the observation in the changes of the normal blood flow to tubes and ovaries.

As the symptoms, signs and physical findings are associated with more than one condition like torted ovarian cyst, renal colic or appendicitis if on the right side, the main diagnosis is often established at laparoscopy or laparotomy. So ours too had pre surgery diagnosis of twisted ovarian cyst.

The management consists of early diagnosis and surgery in form of salpingectomy either by open / laparoscopic route; the latter is safe only in the first trimester of pregnancy. If torsion is of recent onset; it can be untwisted and preserved. Salpingectomy is best for a tube that is beyond recovery. This measure has less likelihood of the escape of embolus from ovarian vessels, which is a feared complication at untwisting.

In this case of concern, laparotomy as a management was suitable because of the enlarged uterine size corresponded to 32 weeks; salpingectomy was ideal for the gangrenous fallopian tube as a result of torsion. ${ }^{7}$

It is luck that the labor was spontaneous and at term. This indicated proper form of management by not touching the uterus. Many of us could have been inclined for caesarean delivery at the time of laparotomy. However the chances of abdominal wound infection could have been reduced possibly with earlier surgical intervention.

\section{Conclusion}

Fallopian tube torsion is uncommon and more uncommon during pregnancy. Therefore, this should be kept in the differential diagnosis of acute abdomen in pregnancy, that might warrant a prompt management in view of better postoperative well being for both mother and the fetus.

\section{Reference:}

1. Nanda S, Walecha N, Singh R.B. Fallopian tube torsion: A case series. Journal of Gynecologic Surgery 22(4):157

2. Bika O, Khan R.S. Torsion of a hydrosalpinx in pregnancy: An unusual cause of acute abdomen in pregnancy. http:/ www.Sexualhealthmatters_.com/v5iss $1 /$ article4.html_

3. Phupong V, Intharasakda P. Twisted fallopian tube in pregnancy: a case report. BMC Pregnancy Childbirth 2001; 1(1):5.

4. Yalcin OT, Hassa H, Zeytinoglu S, Isiksoy S. Isolated torsion of fallopian tube during pregnancy; report of two cases. Eur J Obstet Gynecol Reprod Biol. 1997 Aug;74(2):179-82.

5. Batukan C, Ozgun MT, Turkyilmaz C, Tayyar M. Isolated torsion of the fallopian tube during pregnancy: a case report. J Reprod Med 2007 Aug; 52(8):745-7.

6. Origoni M, Cavoretto P, Conti E, Ferrari A. Isolated tubal torsion in pregnancy. Eur J Obstet Gynecol Reprod Biol. 2009 Jun 1. [Epub ahead of print]

7. Wang PH, Yuan CC, Chao HT, Shu LP, Lai CR.Isolated tubal torsion managed laparoscopically. J Am Assoc Gynecol Laparosc 2000 Aug; 7(3):423-7. 